\title{
P04-7-17 Poster session
}

\section{Heterodimerization of two pore domain K+ channel TASK1 and TALK2 in HEK293 heterologous expression systems}

\author{
Yoshiaki Suzuki, Kanako Tsutsumi, Tatsuya Miyamoto, Hisao Yamamura, Yuji Imaizumi \\ Department of Molecular and Cellular Pharmacology, Graduate School of Parmaceutical Sciences, Nagoya City \\ University, Japan
}

Two-pore-domain $\mathrm{K}+(\mathrm{K} 2 \mathrm{P})$ channels sense a wide variety of stimuli such as mechanical stress, inhalational anesthetics, and changes in extracellular $\mathrm{pH}$ or temperature. The $\mathrm{K} 2 \mathrm{P}$ channel activity forms a background $\mathrm{K}+$ current and, thereby, contributes to resting membrane potentials. Six subfamilies including fifteen subtypes of K2P channels have been identified. Each K2P channel molecule with two pores consists of a homodimer of each subtype. In addition, a few heterodimers mainly within the same subfamilies have been found recently. In the present study, the possibility of heterodimerization between TASK1 (TWIK-Related Acid-Sensitive K+ channel) and TALK2 (TWIK-Related Alkaline $\mathrm{pH}$-Activated $\mathrm{K}+$ channel) was examined. These channels belong to separate subfamilies and show extremely different channel properties. Surprisingly, single molecular imaging analyses in this study using a total internal reflection microscope suggested the heterodimerization of TASK1 and TALK2 in a pancreatic cell line, QGP-1. This heterodimer was also detected using a bimolecular fluorescence complementation assay in a HEK293 heterologous expression system. Fluorescence resonance energy transfer analyses showed that the affinity between TASK1 and TALK2 appeared to be close to those of homodimers. Whole-cell patch-clamp recordings revealed that TASK1 currents in HEK293 cells were significantly attenuated by co-expression of a dominant-negative form of TALK2 in comparison with that of wild-type TALK2. The sensitivities of TASK1-TALK2 tandem constructs to extracellular $\mathrm{pH}$ and halothane were characterized as a unique hybrid of TASK1 and TALK2. These results suggested that heterodimerization of TASK1 and TALK2 provides cells with the ability to make multiple responses to a variety of physiological and pharmacological stimuli. 